\title{
Positional therapy is a valuable treatment alternative for obstructive sleep apnea
}

\author{
Arie Oksenberg, Natan Gadoth \\ Sleep Disorders Unit, Loewenstein Hospital-Rehabilitation Center, Raanana, Israel
}

\section{TO THE EDITOR}

We read with interest the commentary manuscript on "Therapeutic Approaches of obstructive sleep apnea in China". ${ }^{[1]}$ This is an informative article summarizing how obstructive sleep apnea (OSA) is being treated in China. As in other countries, continuous positive airway pressure (CPAP) is the treatment of choice for moderate to severe OSA patients. The authors also described the use of oral appliance and mentioned the role of upper airway (UA) surgical procedures in the treatment of OSA. In addition, they correctly mentioned UA muscle training and hypoglossal nerve stimulation as alternative therapies for OSA. Unfortunately, the role of Positional Therapy (PT), that is, the avoidance of the supine posture during sleep in the treatment of OSA was not described. The authors mention in a single phrase "sleep position adjustment" among the effective therapeutic approaches but fail to provide further information on this mode of therapy.

Dr. Arie Oksenberg, Sleep Disorders Unit Loewenstein Hospital - Rehabilitation

Center, Raanana, Israel

E-mail: arieo@clalit.org.il

\begin{tabular}{|l|}
\hline Access this article online \\
\hline Website: \\
www.intern-med.com \\
\hline DOI: \\
10.2478/jtim-2019-0016 \\
\hline Quick Response Code: \\
\hline \\
\hline \\
\hline
\end{tabular}

We do not know the reason for this omission and we do not know how frequently PT is used in China, but it is important to realize that PT is a valuable therapeutic option for many OSA patients.

People with OSA comprise a heterogeneous group of patients, which are clearly not alike. Based on the effect of body posture positional patients (PP) who present breathing abnormalities only (isolated) or mainly (predominantly) in the supine posture (supine AHI at least double the lateral AHI) on OSA, those patients are divided into and non-positional patients (NPP) who have many breathing abnormalities in both lateral and supine postures (supine AHI less than double the lateral AHI). Compared to NPP, PP are thinner, somehow younger, have significantly less severe breathing abnormalities during sleep, enjoy a better sleep quality and are less sleepy during daytime. ${ }^{[2]}$ This phenotype distinction between PP and NPP is crucial because PP may obtain substantial benefit from PT, since for them the severity of the disease is totally dependent on the sleep time spent or not spent in the supine posture.

The prevalence of PP determined in a sleep unit is about $56 \%$ and even higher for mild to moderate OSA (ranging from $65 \%$ to 69\%). ${ }^{[2]}$ In the Asian OSA patients, the prevalence of PP is even higher, reaching nearly $75 \%$, and for mild OSA, as many as $87 \%$ are PP. ${ }^{[3]}$ Therefore, the majority of Asian as well and non-Asian OSA patients are PP. Lately, a Swiss study assessed the prevalence and predictors of positional OSA in 1719 middle-older subjects of the general population and showed that $53 \%$ were PP. $71.2 \%$ had an Apnea Hypopnea Index $\geq 5$, among them $75 \%$ were PP $(76 \%$ in women and $74 \%$ in men) and $36 \%$ had breathing abnormalities only in the supine posture. ${ }^{[4]}$ Thus, probably after obesity, positional or supine-related OSA is the most dominant phenotype of adult OSA.

In the past, PT was a treatment with low adherence, but it has been improving significantly with the use of a new generation of devices, which appear much more comfortable amenable and effective for PP. ${ }^{[5]}$ 
Information on the use and adherence of PT in the Chinese OSA patients is highly needed.

\section{Conflict of Interest}

The authors declare no conflict of interest.

\section{REFERENCES}

1. Li WY, Wang W. Therapeutic approaches of obstructive apnea in China. J Transl Int Med 2018; 6: 16-20.

2. Oksenberg A, Silverberg DS, Arons E, Radwan H. Positional vs nonpositional obstructive sleep apnea patients. Anthropomorphic, nocturnal polysomnographic and multiple sleep latency test data. Chest 1997; 112: 629-39.

3. Mo JH, Lee CH, Rhee CS, Yoon IY, Kim JW. Positional dependency in Asian patients with obstructive sleep apnea and its implication for hypertension. Arch Otolaryngol Head Neck Surg 2011; 137: 786-90.

4. Heinzer R, Petitpierre NJ, Marti-Soler H, Haba-Rubio J. Prevalence and characteristics of positional sleep apnea in the HypnoLaus population based-cohort. Sleep Med 2018; 48:157-62.

5. Ravesloot MJ, White D, Heinzer R, Oksenberg A, Pépin JL. Efficacy of the New Generation of Devices for Positional Therapy for Patients with Positional Obstructive Sleep Apnea: A Systematic Review of the Literature and Meta-Analysis. J Clin Sleep Med 2017;13: 813-24.

How to cite this article: Oksenberg A, Gadoth N. Positional therapy is a valuable treatment alternative for obstructive sleep apnea. J Transl Int Med 2019; 7: 84-5. 